\title{
DESIGN OF OPTIMAL CONDUCTANCE PROBES FOR TWO-PHASE FLOW TOMOGRAPHY AND LIQUID HOLDUP: APPLICATION TO THE DETERMINATION OF THE AVERAGE VOID FRACTION IN A REGION
}

\author{
JOSÉ LUIS MUÑOZ-COBO, YAGO RIVERA, CÉSAR BERNA \& ALBERTO ESCRIVÁ \\ Instituto de Ingeniería Energética, Universitat Politècnica de València (UPV), Spain
}

\begin{abstract}
The knowledge of the characteristics of two-phase flows is an important issue in a wide variety of engineering applications. The design of optimal conductance probes for two-phase tomography allows the determination of the shape of a rapidly moving wavy film of water under gravity and shear stress forces exerted on the interface by a gas-phase. Obtaining the characteristics of the interfacial waves can be achieved with a proper design of this kind of sensors. Other application of these probes is for holdup, in this case the determination by conductance measurements of the average void fraction inside a region depends on the effective conductivity of the mixture, which depends not only on the void fraction but also on its distribution inside the region, which in general relies on the two-phase flow regime. In this paper we use the expressions developed recently for the relative conductance of two-plate conductance sensors and those developed previously for two-ring sensors, in order to obtain the best probe designs to be used in different applications of annular two-phase flow tomography. In addition, we discuss the best way to optimize these probes to achieve better results and accuracy when measuring the characteristics of the disturbance and ripple waves as height, frequency and amplitude produced in annular two-phase flow. Also, we use these expressions to optimize the design of the sensors for hold up applications, discussing the effect of different void fraction distributions on the effective conductivity and the measured average void fraction. The computed results have been validated with experimental data obtained from different well-known authors in this field. Finally, we discuss the best type of conductance probes to be used for each specific application and the geometric characteristics of its electrodes as width, height, and separation between them.
\end{abstract}

Keywords: two-phase sensor tomography, interfacial wave measurements, holdup, plate conductance probes, sensor design, void fraction determination.

\section{INTRODUCTION}

The knowledge of the characteristics of two-phase flow regimes is an important issue in many two-phase flows devices currently used in the energy and chemical industries [1]-[4]. There are several two-phase flow patterns in horizontal, and vertical pipes, being the annular flow the most relevant one in steam generators of energy plants (nuclear power and combined cycles), refrigeration systems, and pipes used for the transport and production of natural gas [4]-[6]. Each flow pattern in two-phase flow corresponds to a characteristic gas-liquid topology, which defines typical interfaces between the phases of a given flow, as bubbly flow, annular flow, or cap/slug flow. The exact knowledge of the structures as disturbance and ripple waves formed at the interface in annular flow is important for several reasons, the first one is that the heat transfer between the phases depends on the shape and height of these waves, the second is that the pressure drop is highly influenced by these structures. In addition, the third reason is that when the gas phase moves at higher velocity that the liquid phase the shear stress produced at the interface can tear off small drops from the peaks of the disturbance waves reducing its height [7]. Therefore, as observed experimentally [7], [8], over a wide range of liquid and gas superficial velocities the two-phase annular flow pattern in a pipe consists of a thin liquid film moving adjacent to the wall, with a gaseous core 
transporting small drops entrained from the crest of the wavy film. In addition, if the velocity of the gas is smaller than the threshold for drop-entrainment then the drops are not present in the gaseous core.

Several methods have been used in the past to obtain the two-phase flow characteristics, as void fraction distribution in bubbly and cap/slug flow, or wave characteristics in annular flow. To obtain void fraction distribution the most common technique is by conductivity probes with two and three needles [9], [10], and mesh wire sensors [11], while to obtain the average void fraction inside a region one can use the conductance probe method [1], the impedance method [12], [13], and the radiation attenuation method [14]. Finally, to obtain the characteristics of the interfacial waves in annular flow the most common methods are the conductance methods, [1], [15] and the optical ones as the optical laser-based measurement technique, also known as Planar Laser-Induced Fluorescence (PLIF) [16].

The main goal of this paper is to describe how to obtain the best design of the conductance probes, which are used for two-phase tomography mainly of annular flows and holdup applications as determining the average liquid fraction or the average void faction inside a pipe containing a two-phase flow mixture.

The paper has been organized as follows, Section 2 introduces the conductance probe method, presenting in Section 2.1 the expressions for the calculation of the relative conductance in ring and plate conductance sensors. In Section 2.2 we show the expressions used for holdup applications using two-ring electrodes. Then, in Section 2.3 we show how to obtain the error factor used for the design of conductance probes, and the useful range of film thicknesses that we can measure with a conductance probe. Sections 3.1 and 3.2 are devoted to the comparison of the results obtained with different sensor designs of plate probes, while in Section 3.3 we study the use of the conductance sensors for holdup and void fraction determination. Finally, in Section 4 we give the main conclusions of the paper.

\section{THE CONDUCTANCE PROBE METHOD}

In this section we describe the main characteristics of the conductance probe method. It is well known that the measured electrical impedance between a pair of electrodes submerged in a conducting fluid as tap water is essentially resistive when the frequency of the exciting a.c. signal of the emitting electrode is in the range from 10 to $100 \mathrm{kHz}$, in this range of frequencies the impedance methods are known as conductance methods. Two types of electrodes, ring, and plate have been used in conductance probes to measure the film thickness of the conducting fluid [2], [15], [17]. In this section we will study the performance of flush mounted electrodes to determine the best probe designs to have good spatial resolution and sensitivity to perform measurement of the film thickness.

\subsection{The conductance probe method with plate and ring electrodes}

The two-ring conductance probe consist of two ring shape electrodes mounted along the inner circumference of the pipe, this type of electrodes has been studied by Tsochatzidis et al. [2] and Fossa [17]. This kind of conductance probes provides good results to obtain the void fraction in uniformly dispersed bubbly flow regime and even in non-homogeneous bubbly flow as discussed recently by Muñoz-Cobo et al. [1]. The two-plate conductance probe consists in two flush mounted plated electrodes, subjected to a high frequency a.c. electrical excitation with frequencies ranging from 10 to $100 \mathrm{kHz}$, at these frequencies the electric impedance through the conducting liquid is essentially resistive. To calibrate this type of electrodes, to measure film thickness, a set of dielectric cylinders of different radius $R_{\text {in }}$ are mounted inside the pipe as displayed at Fig. 1(a). The probe conductance defined as the ratio 
$G=I / \delta \phi$, is then measured being I the electric intensity circulating through the conducting fluid (electrolyte), and $\delta \phi$ the average electric-potential difference between the emitter and receiver electrodes of the two-ring or two-plate probes i.e., $\delta \phi=\left\langle\phi_{E}\right\rangle-\left\langle\phi_{R}\right\rangle$, which depends on the film thickness $\delta$ of the conducting fluid. If this thickness is very small, then the conductance is also small because of the electric resistance to the pass of ions through the electrolyte is large. Normally, people use the non-dimensional conductance $G^{*}=G /\left(\sigma_{w} l\right)$, which is defined as the ratio of the conductance and the product of the conducting fluid conductivity $\sigma_{w}$ and the characteristic length $l$ of the sensor.

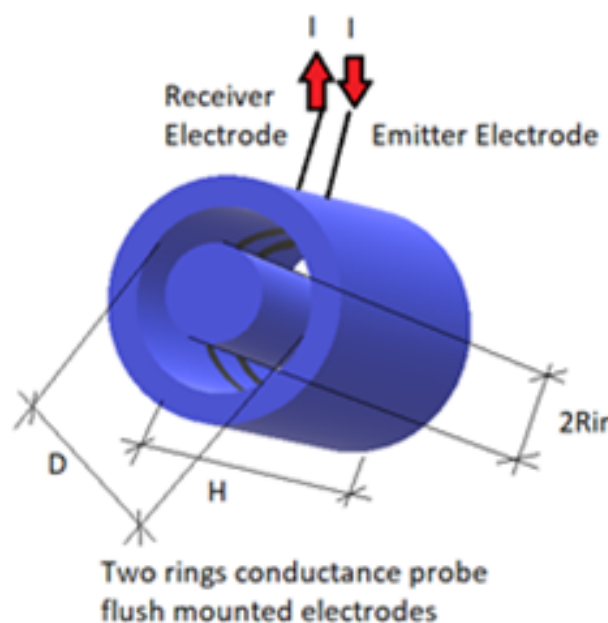

(a)

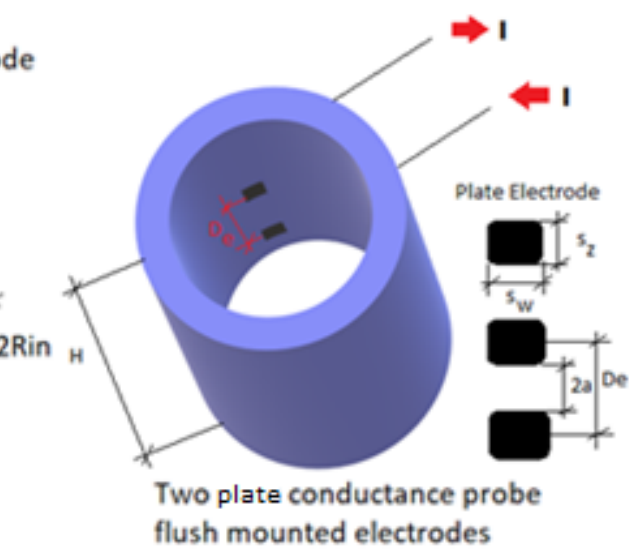

(b)

Figure 1: (a) Two-ring conductance probe with inner dielectric calibration cylinder; and (b) Two plate conductance probe displaying the geometrics characteristics of the electrodes.

The geometric characteristics of a two-electrode conductance sensor are defined by the following geometric lengths, $2 a$, which is the distance between the electrodes, $D_{e}$ that is the distance between the electrode centres, $s_{z}$ that is the height of each electrode in the axial direction and $s_{w}$ that is the width of the electrodes.

However, to present and compare the experiments of different authors with theory, it is convenient due to the variations of the conductivity from one experiment to another, to normalize the non-dimensional conductance $G^{*}$ with the conductance obtained when the pipe is full of water $G_{\max }^{*}$, in this way it is defined the relative conductance $G_{r e l}^{*}$. If one assumes that the sensor probe is located at the centre of the pipe, then the relative conductance expressions for a two-ring conductance sensor obtained by Tsochatzidis et al. [1], [2], and for a two-plate conductance sensor deduced by Muñoz-Cobo et al. [2], can be unified into the following equation:

$$
G_{r e l}^{*}=\frac{G^{*}}{G_{m a x}^{*}}=\frac{C_{1}^{\prime} \sum_{n=0}^{\infty} \frac{b_{n}^{2}}{(2 n+1)^{3}} \frac{I_{0}\left(\gamma_{n} R\right)}{I_{1}\left(\gamma_{n} R\right)}+C_{2}^{\prime} \sum_{n=0}^{\infty} \sum_{m=1}^{\infty} \frac{e_{m, n}^{2}}{(2 n+1)^{3} m} \frac{I_{m}\left(\gamma_{n} R\right)}{I_{m}^{\prime}\left(\gamma_{n} R\right)}}{C_{1}^{\prime} \sum_{n=0}^{\infty} \frac{b_{n}^{2}}{(2 n+1)^{3}} f\left(\gamma_{n} R_{i n}, \gamma_{n} R\right)+C_{2}^{\prime} \sum_{n=0}^{\infty} \sum_{m=1}^{\infty} \frac{e_{m, n}^{2}}{(2 n+1)^{3} m} f_{m}\left(\gamma_{n} R_{i n}, \gamma_{n} R\right)},
$$


being $C_{1}^{\prime}=\frac{\Delta \theta^{2}}{4}, C_{2}^{\prime}=\left\{\begin{array}{c}1 \text { for two }- \text { plate electrodes } \\ 0 \text { for two }- \text { ring electrodes }\end{array}, \gamma_{n}=\frac{(2 n+1) \pi}{H}\right.$, and $\Delta \theta=\frac{s_{w}}{R}$. In addition, we have defined the following quantities:

$$
e_{m, n}=b_{n} \sin \left(\frac{m \Delta \theta}{2}\right) \text { with } b_{n}=\cos \left(\gamma_{n} \frac{D_{e}+s_{z}}{2}\right)-\cos \left(\gamma_{n} \frac{D_{e}-s_{z}}{2}\right) \text {, }
$$

where the functions $f\left(\gamma_{n} R_{i n}, \gamma_{n} R\right)$, and $f_{m}\left(\gamma_{n} R_{i n}, \gamma_{n} R\right)$, which appear in the denominator of eqn (1) are given by the expressions:

$$
\begin{gathered}
f\left(\gamma_{n} R_{i n}, \gamma_{n} R\right)=\frac{I_{0}\left(\gamma_{n} R\right)}{I_{1}\left(\gamma_{n} R\right)}\left\{\frac{1+a_{r}\left(\gamma_{n} R_{i n}\right) \frac{K_{0}\left(\gamma_{n} R\right)}{I_{0}\left(\gamma_{n} R\right)}}{1-a_{r}\left(\gamma_{n} R_{i n}\right) \frac{K_{1}\left(\gamma_{n} R\right)}{I_{1}\left(\gamma_{n} R\right)}}\right\} \text { with } a_{r}\left(\gamma_{n} R_{i n}\right)=\frac{I_{1}\left(\gamma_{n} R_{i n}\right)}{K_{1}\left(\gamma_{n} R_{i n}\right)}, \\
f_{m}\left(\gamma_{n} R_{i n}, \gamma_{n} R\right)=\frac{I_{m}\left(\gamma_{n} R\right)-a_{m, n}\left(\gamma_{n} R_{i n}\right) K_{m}\left(\gamma_{n} R\right)}{I_{m}^{\prime}\left(\gamma_{n} R\right)-a_{m, n}\left(\gamma_{n} R_{i n}\right) K_{m}^{\prime}\left(\gamma_{n} R\right)} \text { with } a_{m, n}\left(\gamma_{n} R_{i n}\right)=\frac{I_{m}^{\prime}\left(\gamma_{n} R_{i n}\right)}{K_{m}^{\prime}\left(\gamma_{n} R_{i n}\right)},
\end{gathered}
$$

being $I_{m}(x), K_{m}(x), I_{m}^{\prime}(x), K_{m}^{\prime}(x)$, the first and second class modified Bessel functions of order $\mathrm{m}$ and their derivatives evaluated at $x=\gamma_{n} R_{\text {in }}$ and $x=\gamma_{n} R$.

When both conductance electrodes are parallels between them and to the pipe axis and flush mounted to the pipe surface as displayed at Fig. 2. Then, the relative conductance for a uniform liquid film located between the inner non conducting dielectric cylinder of radius $R_{\text {in }}$ and the pipe inner radius $R$ is given by the Muñoz-Cobo et al. expression [2]:

$$
G_{\text {rel }}^{*}=\frac{G^{*}}{G_{m a x}^{*}}=\frac{C_{1} \sum_{m=1}^{\infty} \frac{a_{m}^{2}}{m^{3}}+\sum_{n=1}^{\infty} \sum_{m=1}^{\infty} \frac{c_{m, n}^{2} I_{m}\left(\gamma_{n}^{\prime} R\right)}{m^{2} I_{m}^{\prime}\left(\gamma_{n}^{\prime} R\right)}}{C_{1} \sum_{m=1}^{\infty} \frac{a_{m}^{2}\left(1+\left(\frac{R_{i n}}{R}\right)^{2 m}\right)}{m^{3}\left(1-\left(\frac{R_{i n}}{R}\right)^{2 m}\right)}+\sum_{n=1}^{\infty} \sum_{m=1}^{\infty} \frac{c_{m, n}^{2}}{m^{2} n^{3}} f_{m}\left(\gamma_{n}^{\prime} R_{i n}, \gamma_{n}^{\prime} R\right)}
$$

being $C_{1}$ a constant, which depends on the geometric characteristics of the sensor and the pipe, and $\gamma_{n}^{\prime}$ a constant that depends on the harmonic order $\mathrm{n}$ :

$$
C_{1}=\frac{2 \pi^{3} s_{Z}^{2} R}{H^{3}} \text { and } \gamma_{n}^{\prime}=\frac{2 n \pi}{H}
$$

Finally, the constant $c_{m, n}$ is given by the expression:

$$
c_{m, n}=a_{m}\left(\theta_{1}, \theta_{2}\right) \sin \left(\frac{\gamma_{n}^{\prime} s_{z}}{2}\right), \quad \text { with } a_{m}\left(\theta_{1}, \theta_{2}\right)=\cos \left(m \theta_{2}\right)-\cos \left(m \theta_{1}\right),
$$

where, $\theta_{1}$ and $\theta_{2}$ denote the initial and final azimuthal coordinates of the emitter electrode, where it has been assumed that the azimuthal coordinates of the receiver electrode are, $-\theta_{1}$ and $-\theta_{2}$.

2.2 The relative conductance for hold up applications

When we have a two-phase flow mixture with average void fraction $\alpha$ inside the region of the pipe covered by the sensor and we denote by $G_{\alpha}^{*}=G_{\alpha} /\left(\sigma_{e f f} \pi D\right)$, the non-dimensional conductance of the pipe full of the two-phase mixture, being $\sigma_{e f f}$ the effective electrical 
conductivity of the mixture. In addition, we denote by $G_{\max }^{*}=G_{\max } /\left(\sigma_{w} \pi D\right)$ to the nondimensional sensor conductance when the pipe is full of the liquid phase with electrical conductivity $\sigma_{w}$. Then if we use a two-ring electrode sensor with conductance given by the expression [1], [2]:

$$
G_{\max }^{*}=\frac{G_{\max }}{\sigma_{w} \pi D}=\frac{\pi^{3}}{8}\left(\frac{s_{z}}{\mathrm{H}}\right)^{2} \frac{1}{\sum_{n=0}^{\infty} \frac{b_{n}^{2}}{(2 n+1)^{3}} \frac{I_{o}\left(\gamma_{n} R\right)}{I_{1}\left(\gamma_{n} R\right)}},
$$

being the meaning of the symbols the same ones that in the previous section. Then the conductance ratio of the pipe full of the two-phase mixture and the pipe full of water is given by:

$$
\frac{G_{\alpha}}{G_{\max }}=\frac{G_{\alpha}^{*}}{G_{\max }^{*}} \frac{\sigma_{e f f}}{\sigma_{w}}=\frac{\sigma_{e f f}}{\sigma_{w}}
$$

Eqn (9) has been used by different author to measure the liquid holdup $\alpha_{l}=1-\alpha$. The problem is that to obtain $\alpha$ directly from the relative conductance value, this last value depends not only on the average void fraction but also on its distribution as will be discussed later.

\subsection{The useful range of thicknesses of a conductance probe}

As the film thickness becomes larger the variations $\Delta G^{*}$ in the response of the conductanceprobe to variations $\Delta \delta$ of the film thickness becomes smaller, this means that the range of thicknesses that the probe can measure with good accuracy is limited. For this reason, Coney defined an error factor $f_{\varepsilon}$ as follows [15]:

$$
f_{\varepsilon}=\lim _{\Delta G^{*} \rightarrow 0} \frac{\Delta \delta / \delta}{\Delta G^{*} / G^{*}}=\frac{G^{*}}{\delta} \frac{d \delta}{d G^{*}} .
$$

It is more convenient to express this factor in terms of the relative conductance with respect to the maximum conductance. The maximum conductance value is attained when the pipe is full of water, and the relative conductance $G_{\text {rel }}^{*}$ is defined as the ratio of the conductance for a given annular flow and the value of the conductance for the pipe full of liquid:

$$
G_{r e l}^{*}=\frac{G^{*}}{G_{\max }^{*}}
$$

Dividing the numerator and the denominator of eqn (10) by $G_{\max }^{*}$ and because of the maximum conductance is independent of $\delta$, we obtain the following equation:

$$
f_{\varepsilon}=\frac{G_{r e l}^{*}}{\delta} \frac{d \delta}{d G_{r e l}^{*}}
$$

From eqn (12) it is deduced that the error $\Delta \delta$ obtained in the measurement of the film thickness is related to error $\Delta G_{\text {rel }}^{*}$ obtained in the measurement of the relative conductance by the following approximate expression:

$$
\frac{\Delta \delta}{\delta} \cong f_{\varepsilon} \frac{\Delta G_{r e l}^{*}}{G_{r e l}^{*}}
$$


One of the applications of the error factors $f_{\varepsilon}$ suggested by Coney [15], is to compare the useful ranges of different conductance sensors.

\section{COMPUTED RESULTS FOR DIFFERENTS CONDUCTANCE DESIGNS}

\subsection{Computed results with plate probes of parallel electrodes}

Fig. 2 displays the results obtained for $G_{r e l}^{*}$ versus the water film thickness $\delta$ for two parallel plate electrodes when we change the width of the electrodes. The radius of the pipe was fixed at $\mathrm{R}=25.4 \mathrm{~mm}$, and the distance between the electrodes was also fixed at $2 a=2 \mathrm{~mm}$. It is observed at Fig. 2 that when the width of the electrodes become smaller maintaining the same length at $s_{z}=2.88 \mathrm{~mm}$, then the relative conductance saturates for smaller film thicknesses i.e. it attains values which are closer to its maximum for smaller film thicknesses.

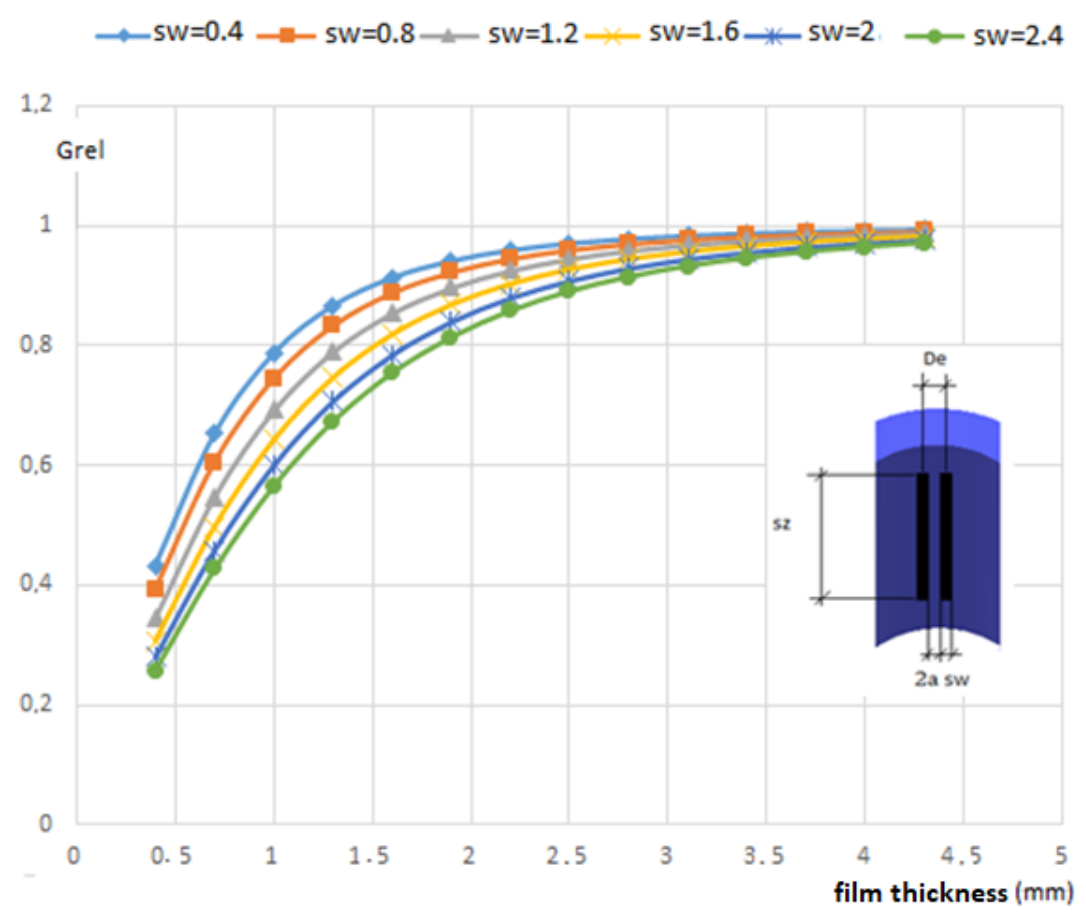

Figure 2: $\quad G_{\text {rel }}^{*}$ versus the film thickness $\delta(\mathrm{mm})$ for a sensor design with two parallel plate geometry and dimensions $s_{z}=2.88 \mathrm{~mm}, a=1 \mathrm{~mm}, R=25.4 \mathrm{~mm}$, for different values of $s_{w}$ in $\mathrm{mm}$.

Therefore, when the conductance is close to its saturation, then small changes in the conductance value produces big changes in the film thickness, and the errors in the film thickness measurements tend to increase. For this same case, we have computed the error factor $f_{\varepsilon}$, by increasing the film thickness of the previous cases in $\Delta \delta=0.05 \mathrm{~mm}$, and obtaining the corresponding increment in the film conductance $\Delta G_{r e l}^{*}$, and the applying eqn (13) to obtain the error factor values. The results are displayed in Fig. 3. 


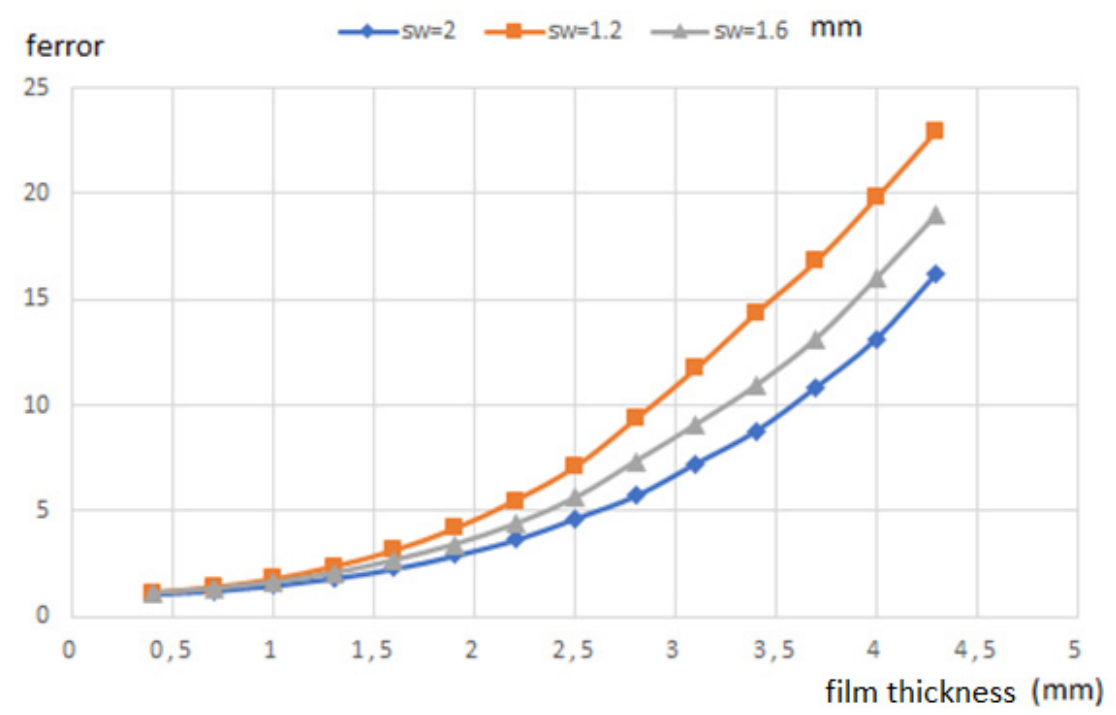

Figure 3: $\quad f_{\varepsilon}$ (error factor) versus the film thickness $\delta(\mathrm{mm})$ for a sensor design with two parallel plates geometry, and dimensions $s_{z}=2.88 \mathrm{~mm}, a=1 \mathrm{~mm}, R=$ $25.4 \mathrm{~mm}$, for different values of $s_{w}$ in $\mathrm{mm}$.

Fig. 3 tells us two things, the first is that when the film thickness increases the error factor also increases, this means that the conductance probe loss progressively its sensitivity when the film becomes thicker. Therefore, small errors in the conductance produce large errors in measuring the film thickness for large thicknesses of the conducting film. The solution to ameliorate this situation is to make larger the width of the electrodes. Increasing the size of the electrodes from $1.2 \mathrm{~mm}$ to $2 \mathrm{~mm}$ reduces the error factor at $1.9 \mathrm{~mm}$ from 4.16 to 2.91 .

\subsection{Computed results with plate probes in the flow direction}

In two-phase flow tomography of annular flows, we are interested in measuring the height, shape, and frequency of the different types of waves, which are formed at the interface between the gas and liquid phases. These waves are mainly of two types: the first are the disturbance waves, which are prominent waves with a height that is approximately four times the thickness of the film and that are coherent in both the streamwise direction and circumferentially. The second class are the ripple waves which are not coherent in any direction and are much smaller in size. Also, although less frequents than the previous ones we can encounter the ephemeral waves, which have also large amplitudes and are no coherent. To measure the characteristics of these waves propagating in the streamwise direction we use conductance plate electrodes, as the ones displayed in Fig. 1(b), which should be as small as possible to capture the characteristic details of these waves. The problem is that if the waves become too large in amplitude and the size of the probe is two small the conductance probe can saturate. For this reason, it is convenient to be able to design the geometric characteristics of the probes (size of the electrodes and distance between them), for each specific application. Fig. 4 displays the relative conductance versus the film thickness $\delta$ in mm for plate conductance probes flush mounted in a pipe of $25.4 \mathrm{~mm}$ of radius. 


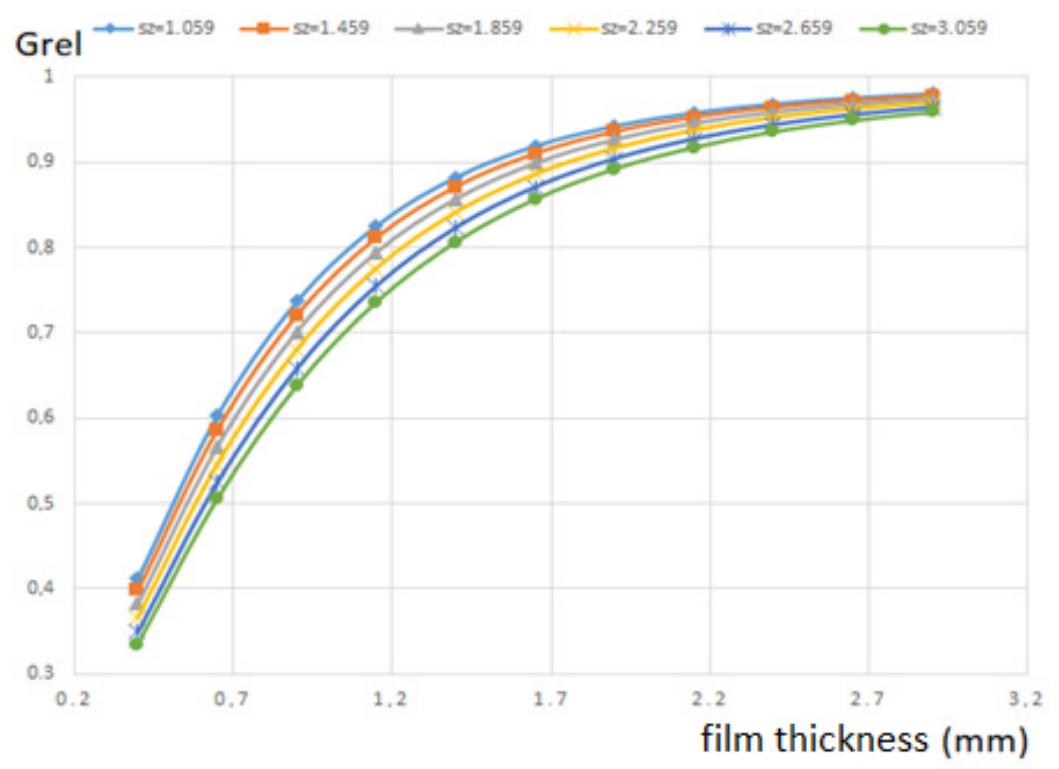

Figure 4: $\quad G_{\text {rel }}^{*}$ versus the film thickness $\delta(\mathrm{mm})$ for a sensor design with two plate electrodes along the flow direction with geometry, and dimensions $s_{w}=2.6586$ $\mathrm{mm}, \mathrm{a}=1 \mathrm{~mm}, \mathrm{R}=25.4 \mathrm{~mm}$, for different values of $s_{z}$ in $\mathrm{mm}$.

It is observed in Fig. 4 that the conductance probe attains earlier the saturation for smaller film thicknesses as the electrode length $s_{z}$ in the streamwise direction becomes smaller. The considered electrode geometries with distances $D_{e}=2 a+s_{z}$ between the centre of the electrodes ranging from $3 \mathrm{~mm}$ to $5 \mathrm{~mm}$, provide good estimation of the film thickness in the range from 0.2 to $1.7 \mathrm{~mm}$. Although for values of the thickness above $1.5 \mathrm{~mm}$ the errors become larger. Fig. 5 displays the error factors for $s_{z}=1.059 \mathrm{~mm}, s_{z}=1.859 \mathrm{~mm}$, and $s_{z}=$ $3.059 \mathrm{~mm}$, we notice that the error factors increase with the thickness of the film. For the conductance sensors with the smaller electrodes about $1 \mathrm{~mm}$, we have acceptable error factors of 4 below a film thickness of $1.5 \mathrm{~mm}$. However, for the sensor with $s_{z}=3.059 \mathrm{~mm}$ the factor of error is below 4.2 for film thickness smaller than $2 \mathrm{~mm}$.

\subsection{Application of the conductance probe to holdup determination}

The application of two-ring conductance probes to holdup applications allows to obtain the liquid fraction or the void fraction values directly from the relative conductance of the gas liquid mixture to that of the pipe full of the conducting liquid (water). If the void fraction distribution in the mixture is homogeneous then as found by Tshochatzidis [2], the Maxwell equation for the effective conductivity provides the best prediction of the liquid fraction from the relative conductance [1], [2]. However, if the mixture becomes non-homogeneous then Muñoz-Cobo et al. [1] found that the expression that best fitted the experimental data of Yang et al. [12] was the effective medium theory (EMT) in the self-consistent approximation. However, this approximation fails for values of the liquid fraction below 0.86 , as observed in Fig. 6. To try to improve these results we have used the percolation theory in continuous 


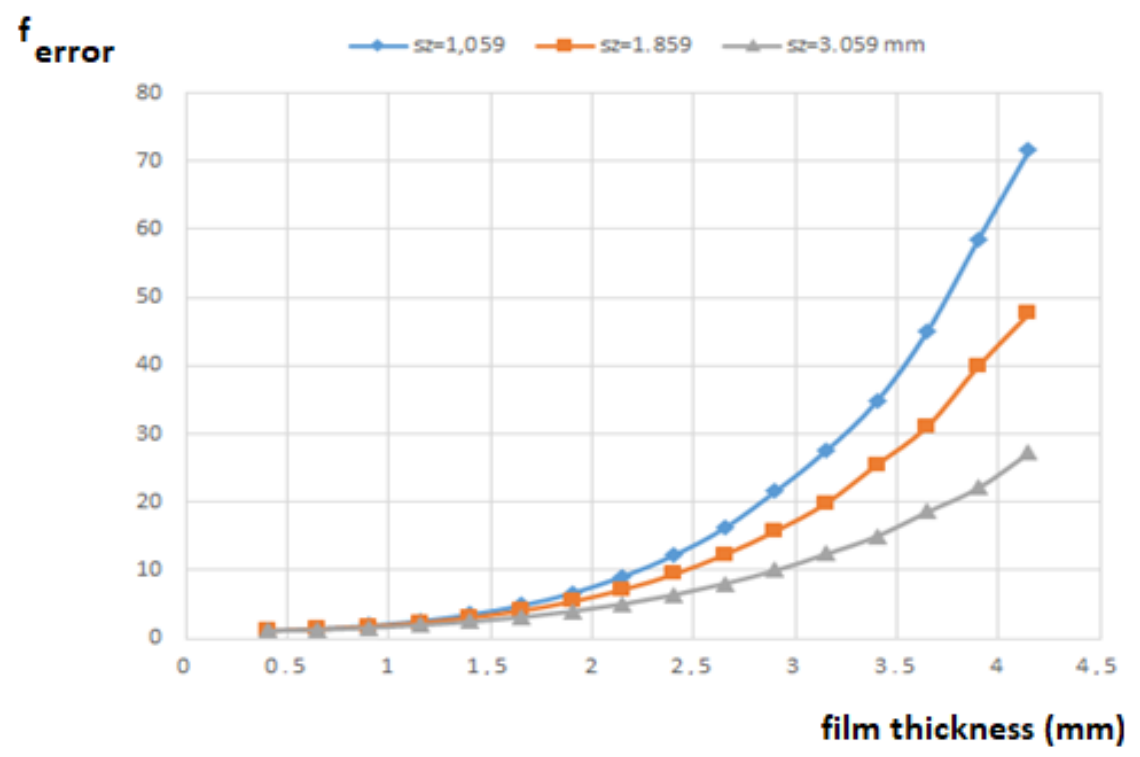

(a)

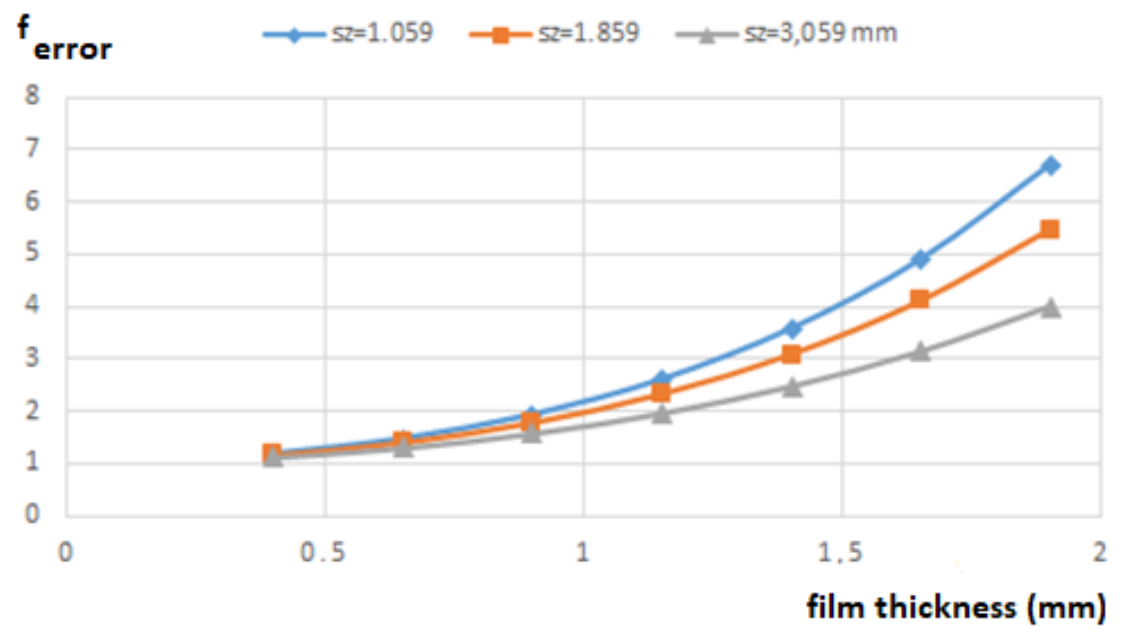

(b)

Figure 5: $\quad$ Error factor $f_{\varepsilon}$ versus the film thickness $\delta(\mathrm{mm})$ for a sensor design with two plate electrodes along the flow direction with geometry, and dimensions $s_{w}=2.6586$ $\mathrm{mm}, \mathrm{a}=1 \mathrm{~mm}, \mathrm{R}=25.4 \mathrm{~mm}$, for different values of $s_{z}$ in $\mathrm{mm}$. (a) For film thickness up to $4.5 \mathrm{~mm}$; and (b) For film thickness up to $2 \mathrm{~mm}$.

media, which provides much better predictions than the previous ones for non-homogeneous bubbly flows as observed in Fig. 6. This theory is especially applicable when the electrical conductivities of both phases of the two-phase mixture differs significantly as happens in the present case where the conductivity of the gas phase is negligible [18], [19]. 


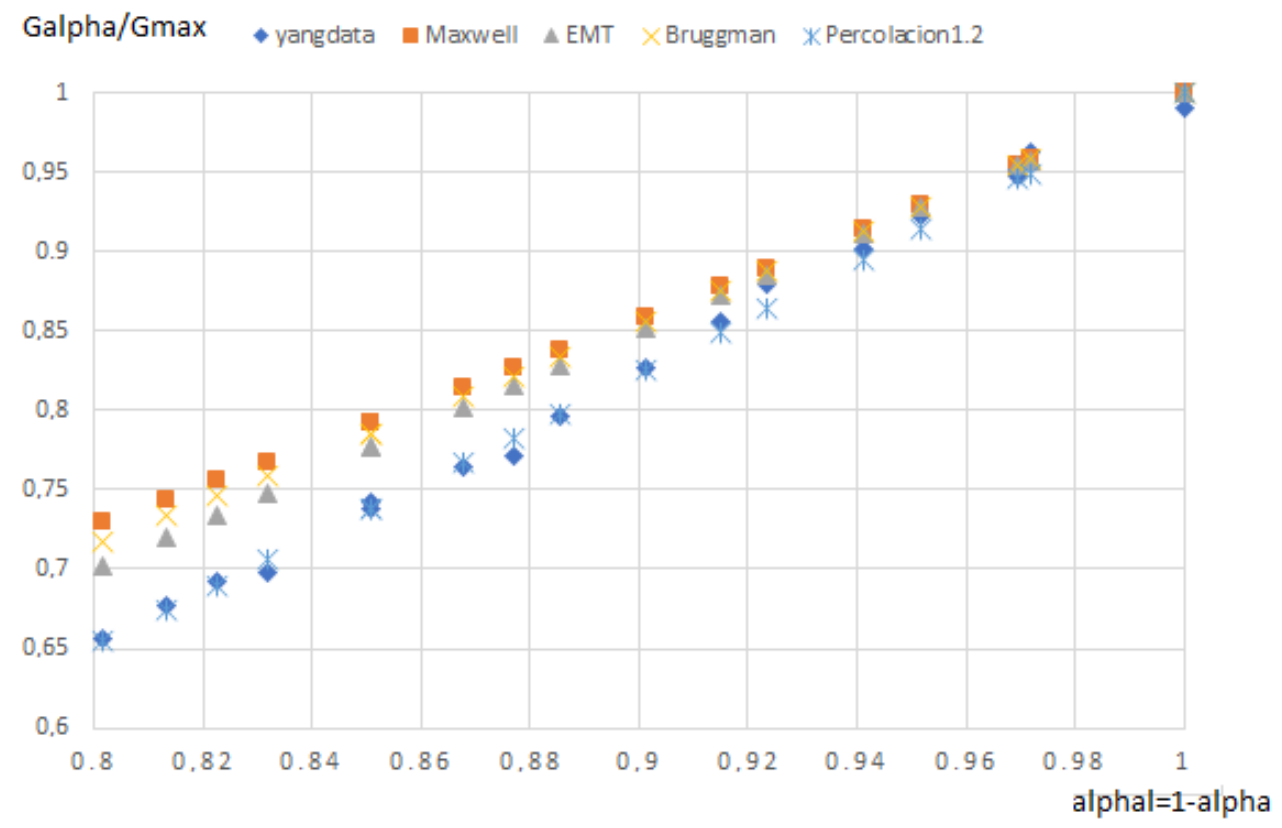

Figure 6: Relative conductance of the two-phase mixture versus the liquid fraction $\alpha_{l}=$ $1-\alpha$, for different models Maxwell (squares), self-consistent EMT (triangles), Bruggman (x), percolation (blue $\mathrm{x}$ with a vertical line) and Yang et al. [12] data (rhomboids).

The percolation theory used to predict the effective conductivity of the two-phase mixture gives the following expression for the effective conductivity [18], [19]:

$$
\sigma_{e f f}=\sigma_{w}\left(\frac{\alpha_{l}-\alpha_{c}}{1-\alpha_{c}}\right)^{\mu} \text { for } \alpha_{l}>\alpha_{c} \text { and } \sigma_{e f f}=0 \text { for } \alpha_{l}<\alpha_{c} \text {, }
$$

where $\alpha_{c}$ is the percolation threshold for the liquid fraction of the conducting phase that is equal to $1 / 3, \alpha_{l}$ is the volume fraction of the conducting phase, finally $\mu$ is the critical exponent. The calculations of the effective conductivity using the percolation model have been performed with a percolation threshold $\alpha_{l}=1 / 3$, and a critical exponent $\mu=1.2$.

Finally Fig. 7 displays the relative conductance versus the liquid fraction computed with the percolation model for critical exponents of $1.15,1.2$ and 1.25 we see that the three values match the experimental data, but the critical exponent of 1.25 gives results which are a little lower than the experimental ones for the lower liquid fractions.

\section{CONCLUSIONS}

In this paper we have discussed how to obtain the best design of the conductance probes, which are used for two-phase tomography mainly of annular flows and hold up applications as determining the average liquid fraction or the average void faction inside a pipe containing a two-phase flow mixture. We have performed the calculation of the useful range of the conductance probes by means of the error factor that gives as expressed by eqn (13), how is 


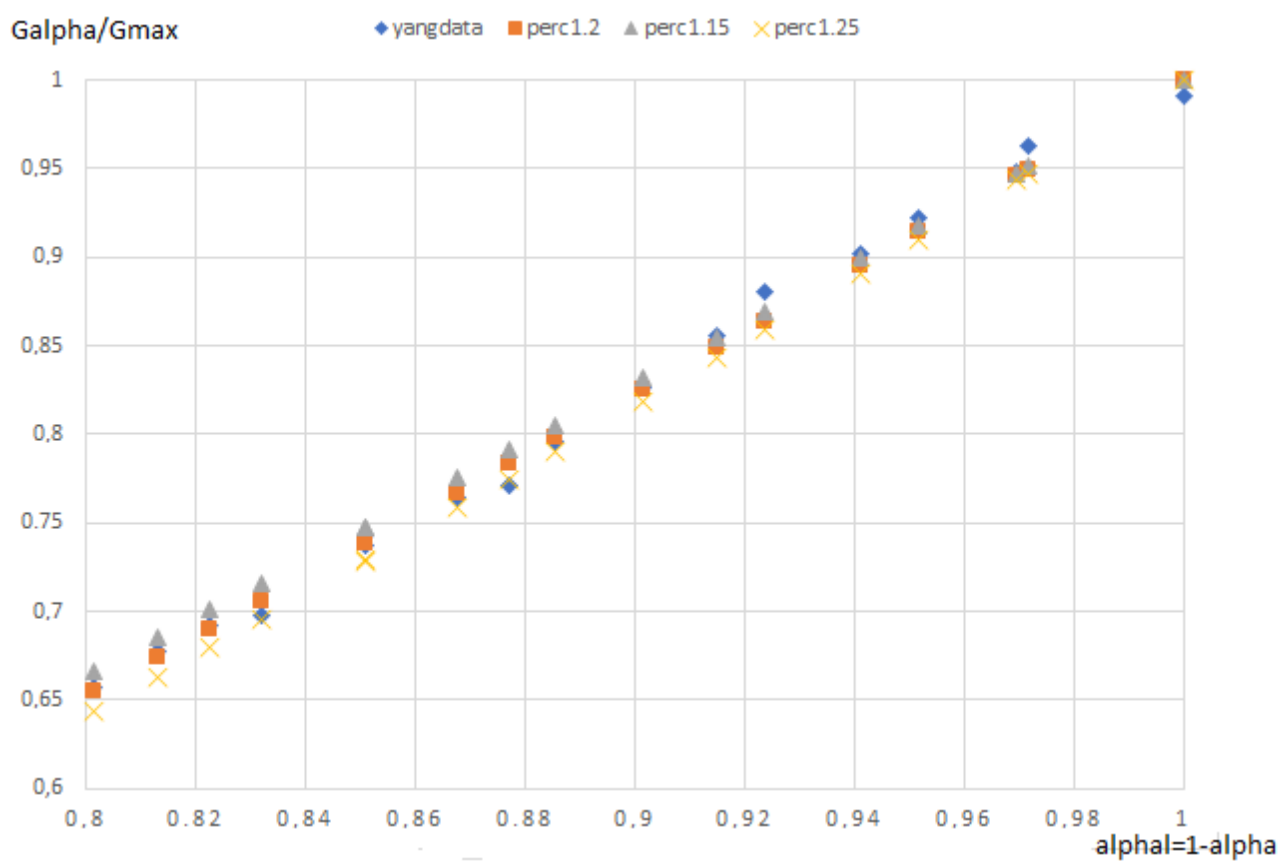

Figure 7: Relative conductance versus the liquid fraction for Yang experiments [12], and the percolation model computed with the critical exponents of 1.15, 1.2 and 1.25.

transformed the relative error in the conductance to the relative error in the film thickness. Because the conductance depends on the conductivity and the conductivity changes with the temperature, these error factors give us if the conductivity is maintained within a given error for instance 4\%, the error that we can have when measuring the film thickness. For instance, if the error factor for a conductance sensor design is 4 , and the conductivity is maintained constant within a $4 \%$, then we will have an error in $\delta$ of $16 \%$. If we want a tolerance for $\delta$ smaller than $10 \%$, then with the curve of Fig. 5 , we shall use a sensor design with $s_{Z}=3 \mathrm{~mm}$, with this height of the electrodes and the geometry given in the caption of Fig. 5, we are limited to measure film thickness smaller than $1.4 \mathrm{~mm}$, to have a tolerance smaller than $10 \%$.

Another important issue studied in this paper is the prediction of the liquid holdup or the void fraction when the bubbly flow is not homogeneous, we have seen that in this case the best prediction of Yang et al. [12] data is obtained with the percolation theory of 3D continuous systems, in this case the threshold and the critical exponents are computed by Monte-Carlo although many authors [18] recommend considering the critical exponent $\mu$ as a fitting parameter. The calculations of Fig. 6 were performed with a critical exponent value of $\mu=1.2$.

\section{ACKNOWLEDGEMENT}

The authors of this paper are indebted to the financial support received from Spain Ministry of Science and Technology contract number ENE2016-79489-C2-1-P (EXMOTRANSIN), of the Plan Nacional de I + D. 


\section{REFERENCES}

[1] Muñoz-Cobo, J.L., Rivera, Y., Berna, C. \& Escrivá, A., Analysis of conductance probes for two-phase flow and holdup applications. Sensors, 20, p. 7042.

DOI: 10.3390/s20247, 2020.

[2] Tsochatzidis, N.A., Karapantsios, T.D., Kostoglou, M.V. \& Karabelas, A.J., A conductance probe for measuring liquid fraction in pipes and packed beds. International Journal of Multiphase Flow, 18(5), pp. 653-667, 1992.

[3] Collier, J.G. \& Thome, J.R., Convective Boiling and Condensation, Oxford Engineering Science Series, Editorial Clarendon Press, pp. 1-16, 1994.

[4] Belt, R.J., Vant't Westende, J.M.C., Prasser, H.M. \& Portela, L.M., Time and spatially resolved measurements of interfacial waves in vertical annular flow. International Journal of Multiphase Flow, 36, pp. 570-587, 2010.

[5] Zhao, Y., Markides, C. \& Hewitt, G.F., Disturbance wave development in two-phase gas-liquid upward vertical annular flow. International Journal of Multiphase Flow, 55, pp. 111-129, 2013.

[6] Rivera, Y., Muñoz-Cobo, J.L., Cuadros, J.L., Berna, C. \& Escrivá, A., Experimental study of the effects produced by the changes of the liquid and gas superficial velocities and the surface tension on the interfacial waves and the film thickness in annular concurrent upward vertical flows. Experimental Thermal and Fluid Science, 120, p. 110224, 2021.

[7] Berna, C., Escrivá, A., Muñoz-Cobo, J.L. \& Herranz, L., Review of droplet entrainment in annular flow: Interfacial waves and onset of entrainment. Progress in Nuclear Energy, 74, pp. 14-43, 2014.

[8] Lopes, J.C.B. \& Dukler, A.E., Dopler entrainment in vertical annular flow and its contribution to momentum transfer. AICHE Journal, 32(9), pp. 1500-1515, 1986.

[9] Munoz-Cobo, J.L., Chiva, S., Mendes, S., Monrós, G., Escrivá, A. \& Cuadros, J.L., Development of conductivity sensors for multi-phase flow local measurements at the Polytechnic University of Valencia (UPV) and University Jaume I of Castellon (UJI). Sensors, 17, p. 1077, 2017. DOI: 10.3390/s17051077.

[10] Manera, A., Ozar, B., Paranjape, S., Ishii, M. \& Prasser, H., Comparison between wiremesh sensors and conductive needle-probes for measurements of two-phase flow parameter. Nuclear Engineering and Design, 239, pp. 1718-1724, 2009.

[11] Prasser, H.M., Boettger, A. \& Zschau, J., A new electrode-mesh tomography for gasliquid flows. Flow Measurement and Instrumentation, 9, pp. 111-119, 1998.

[12] Yang, H.C., Kim, D.K. \& Kim, M.H., Void fraction measurement using impedance method. Flow Measurement and Instrumentation, 14, pp. 151-160, 2003.

[13] Andreussi, P., Donfrancesco, A.D. \& Messia, M., An impedance method for the measurement of liquid hold-up in two-phase flow. International Journal of Multiphase Flow, 14, pp. 777-785, 1988.

[14] Jones, O.C. \& Zuber, N., The interrelation between void fraction fluctuations and flow patterns in two-phase flow. International Journal of Multi-Phase Flow, 2, pp. 273306, 1975.

[15] Coney, M.W.E., The theory and application of conductance probes for the measurement of liquid film thickness in two phase flow. Journal of Physics E: Scientific Instruments, 6, pp. 903-910, 1973.

[16] Zadrazil, I., Matar, O.K. \& Markides, C.N., An experimental characterization of downwards gas-liquid annular flow. International Journal of Multiphase Flow, 60, pp. 87-102, 2014. 
[17] Fossa, M., Design, and performance of a conductance probe for measuring the liquid fraction in two-phase gas-liquid flows. Flow Measurement and Instrumentation, 9, pp. 103-109, 1998.

[18] Kovacik, J., Electrical conductivity of two-phase composite materials. Script Materialia, 39(2), pp. 153-157, 1998.

[19] Stauffer, D. \& Aharony, A., Introduction to Percolation Theory, 2nd edn, Taylor and Francis: London, 1992. 